\title{
Deep brain stimulation of the subthalamic nucleus improves sense of well-being in Parkinson's disease
}

\author{
Louise M. McDonald, $\mathrm{PhD}^{1}$, Donna Page, $\mathrm{MSc}^{1,2}$, Leonora Wilkinson, $\mathrm{PhD}^{1,3}$, and Marjan \\ Jahanshahi, PhD $^{1}$ \\ ${ }^{1}$ Sobell Department of Motor Neuroscience and Movement Disorders, UCL Institute of Neurology, \\ Box 146, 33 Queen Square, London WC1N 3BG, UK \\ ${ }^{3}$ Behavioral Neurology Unit, National Institute of Neurological Disorders and Stroke, Bethesda, \\ MD 20892-1430, US
}

\begin{abstract}
Deep brain stimulation of the subthalamic nucleus is an effective treatment for the motor symptoms of Parkinson's disease. Although a range of psychiatric and behavioural problems have been documented following deep brain stimulation, the short-term effects of subthalamic nucleus stimulation on patients' mood have only been investigated in a few studies. Our aim was to compare self-reported mood in Parkinson's patients with deep brain stimulation of the subthalamic nucleus ON versus OFF. Twenty-three Parkinson's patients with bilateral deep brain stimulation of the subthalamic nucleus and 11 unoperated Parkinson's patients completed a mood visual analogue scale twice. Operated patients were tested with deep brain stimulation of the subthalamic nucleus both ON and OFF. All were assessed on medication. The operated Parkinson's group reported feeling significantly better coordinated, stronger and more contented with deep brain stimulation ON compared to OFF. Fourteen of the 16 mood scales changed in a positive direction when deep brain stimulation of the subthalamic nucleus was ON. When changes in motor scores were taken into account, the operated patients still reported feeling better-coordinated, but also less gregarious with stimulation ON. Unoperated Parkinson's patients showed no differences on any of these measures between their two ratings. Short-term changes in deep brain stimulation of the subthalamic nucleus have a small and mostly positive effect on mood, which may be partly related to improvements in motor symptoms. The implications for day-to-day management of patients with deep brain stimulation of the subthalamic nucleus are discussed.
\end{abstract}

\section{Keywords}

Parkinson's disease; deep brain stimulation; subthalamic nucleus; mood; emotion

Correspondence should be addressed to: Professor M. Jahanshahi, Sobell Department of Motor Neuroscience and Movement Disorders (Box 146), Institute of Neurology, 33 Queen Square, London, WC1N 3BG. Telephone: +44 (0)20 34488733 Fax: +44 (0)20 74191860 m.jahanshahi@ucl.ac.uk.

${ }^{2}$ Current address: Ballito Medical Centre, The Well, Albertino Way, Ballito 4420, South Africa

Full financial disclosure of all authors for the past year: None

Author contributions: McDonald: 2A, 2B, 3A, 3B

Page: 1A, 1B, 1C, 2A, 2B, 2C, 3B

Wilkinson: 1B, 1C, 2C, 3B

Jahanshahi: 1A, 1B, 1C, 2A,2C, 3B

Financial disclosure/conflict of interest: This work was supported by a postdoctoral fellowship from Parkinson's UK to LW and an NIH R01 grant (NS40865-02) to MJ. 


\section{Introduction}

Deep brain stimulation of the subthalamic nucleus (STN-DBS) is an effective treatment for the motor symptoms of Parkinson's disease (PD) ${ }^{1,2}$. Psychiatric problems including confusion, hypomania, apathy, depression, suicide, anxiety, impulse control disorders and dopamine dysregulation syndrome have been observed ${ }^{3}$ and inability to recognize facial expressions of fear, anger and sadness has been documented when comparing STN-DBS patients pre- and post-surgery ${ }^{4}$. However, little evidence is available on the effects of stimulation on mood and behaviour ${ }^{5,6}$. Switching STN stimulation ON reportedly reduced fatigue and confusion ${ }^{5,6}$, increased activity and euphoria, decreased sedation and dysphoria ${ }^{7}$ and improved cognitive and affective scores on the Beck Depression Inventory (BDI) ${ }^{8}$. A better understanding of the effects of STN-DBS on a wide range of mood states would help clinicians improve the day-to-day management of PD patients with STN-DBS and provide information to help understand longer-term effects of STN-DBS.

We used a 16 item visual analogue mood rating scale (VAS) developed by Norris ${ }^{9}$ and Bond and Lader ${ }^{10}$ to examine the short-term effects on mood of STN stimulation ON versus OFF. A VAS was chosen because it is easy for participants to understand and complete and is repeatable within the same session. The VAS used here covers a wide range of moods, including most of the basic moods ${ }^{11}$ and has been shown to be sensitive to changes in mood state in response to a range of drugs which are known to affect motor performance ${ }^{9}$. It also includes somatic and alertness items, which may be relevant to STN-DBS. The 16 items loaded onto three psychometrically-validated factors: Alert-Sedated, Contented-

Discontented and Calm-Relaxed in controls and healthy participants who had taken sedative drugs ${ }^{10}$. We predicted that stimulation would significantly improve mood compared to DBS OFF, with no differences when control unoperated PD patients completed the mood VAS twice.

\section{Methods}

\section{Participants}

Participants were PD patients undergoing surgery at the Functional Neurosurgery Unit at the Sobell Department of Motor Neuroscience and Movement Disorders, Institute of Neurology. All operated PD patients had undergone bilateral STN-DBS surgery for PD at least 3 months before the assessment took place (range $3-32 \mathrm{~m}$, median $10 \mathrm{~m}$, mean $12.8 \mathrm{~m} \pm 8.2$ ). Prior to surgery, all were screened using neuropsychological and neuropsychiatric assessments to ensure that there was no evidence of dementia or any major psychiatric illness. After surgery, MRI was used to confirm correct positioning of the electrodes in the sensorimotor section of the STN. In every case, DBS was assessed as effective in improving the motor symptoms of PD by a consultant neurologist, which was confirmed by improvements in UPDRS-III scores. There were no lasting side-effects of surgery in any DBS patient. The control participants were PD patients who were suitable for DBS and on the surgery waiting list at the time of testing. Demographic and clinical data for STN-DBS patients and PD controls are shown in Table 1.

The study was approved by the Joint Ethics Committee of the Institute of Neurology and The National Hospital for Neurology and Neurosurgery. Informed consent was obtained in writing from all participants prior to participation in the study.

\section{Mood ratings}

The VAS consisted of 16 visual analogue items each representing opposite extremes of mood, with the following labels at each end: alert—drowsy, calm—excited, strong—weak, muzzy—clear-headed, well-coordinated—clumsy, lethargic—energetic, contented— 
discontented, troubled - tranquil, mentally slow—quick-witted, tense-relaxed, attentivedreamy, incompetent—proficient, happy—sad, antagonistic—amicable, interested—bored, withdrawn-gregarious. Eight of the 16 scales had the more positive mood at the left end of the scale with eight at the right end. These sub-scales loaded on three factors: AlertnessSedation (alert, strong, clear-headed, well-coordinated, energetic, quick-witted, attentive, proficient, interested); Contented-Discontented (contented, tranquil, happy, amicable, gregarious); Calm-Relaxed (calm, relaxed). For each scale, a line was drawn between each mood state and its opposite and participants rated their current mood by placing a vertical mark on the line. Each mood VAS was measured in $\mathrm{mm}$ from the positive end. Scores ranged from 0 to 100 , with lower scores indicating more positive mood. Participants also rated the effort required and the physical and mental fatigue they experienced when completing a series of other cognitive and motor tests with DBS ON or OFF on three Likert scales with a range from 0 (none) -10 (a great deal).

\section{Procedure}

Participants rated their feelings "AT THE MOMENT" on each VAS. The STN-DBS patients rated themselves once when ON stimulation and once when OFF stimulation, with order counterbalanced across participants.

Stimulation was switched ON or OFF 30 min before testing. All STN-DBS patients were on their usual medication. PD controls rated themselves twice, at least 1.5 hours apart, and were tested on medication. The mood ratings were completed as part of a series of cognitive and motor assessments lasting a total of two hours, which were completed with DBS ON and DBS OFF.

Participants completed the Raven's Advanced Progressive Matrices ${ }^{12}$ and the National Adult Reading Test ${ }^{13}$ to provide estimates of current and premorbid IQ and the Hospital Anxiety and Depression Scales ${ }^{14}$ to assess anxiety and depression. The Starkstein Apathy Scale ${ }^{15}$ was used to assess apathy.

\section{Analysis}

Most VAS items showed a normal distribution, so the two mood ratings for participants were compared using paired t-tests. The scales were also combined into factors which were analysed with paired t-tests. Levodopa equivalent doses were calculated using a formula from Tomlinson et al. ${ }^{16}$ with conversion factors for cabergoline and pergolide added from Williams-Gray et al. ${ }^{17}$ In order to explore the relationship between changes in mood and changes in motor symptoms, an additional analysis of covariance (ANCOVA) was completed using the change in UPDRS-III scores with DBS ON or OFF (on medication) as a covariate.

\section{Results}

The groups were well-matched and the only significant difference between the STN-DBS and control PD groups was that the former had a longer duration of illness $(\mathrm{t}(32)=2.42, \mathrm{p}$ $=.021)$. Within the STN-DBS group, the post-surgery UPDRS-III score ON stimulation and OFF medication was significantly lower than the pre-surgery UPDRS score OFF medication $(\mathrm{t}(15)=5.36, \mathrm{p}<.0001)$, indicating that STN-DBS had been effective in improving motor symptoms. Similarly, UPDRS-III scores with DBS ON and on medication were significantly improved compared to scores with DBS OFF and ON medication $(\mathrm{t}(18)=-4.75, \mathrm{p}<.0001)$. The STN-DBS group also took significantly less medication after surgery $(\mathrm{t}(13)=4.5, \mathrm{p}=$. 001). 


\section{Mood data}

When their stimulation was switched ON, STN-DBS patients reported themselves as significantly better-coordinated $(\mathrm{t}(22)=-5.76, \mathrm{p}<0.0001)$, stronger $(\mathrm{t}(22)=-2.41, \mathrm{p}=$. $025)$ and more content $(t(22)=-2.50, p=.021$; Figure 1a). For the remaining scales, the DBS ON versus OFF effects were not significant. All results, except for amicable and gregarious, were in the direction of more positive mood when stimulation was ON compared to OFF.

Analysis of the STN-DBS patients' mood data by factors showed a significant improvement in Alertness-Sedation $(\mathrm{t}(22)=-2.17, \mathrm{p}=.041)$ and Calmness-Relaxation $(\mathrm{t}(22)=-2.54, \mathrm{p}$ $=.019$ ), indicating that STN-DBS patients were significantly more alert and calmer with stimulation switched ON (Figure 2a). There was no significant change in ContentedDiscontented.

Control PD patients showed no significant changes in any of the individual mood scales or combined factors between their two completions of the mood VAS (Figures $1 b$ and $2 b$ ).

\section{Effort and fatigue}

STN-DBS patients reported that they needed significantly less effort to complete the tests (which included the other cognitive tests mentioned in the methods section) when their stimulation was $\mathrm{ON}(\mathrm{t}(22)=-2.22, \mathrm{p}=.037$; Figure $3 \mathrm{a})$. The ratings of physical fatigue and mental fatigue were no different with DBS ON versus OFF. Control PD patients reported no differences on these measures for their two completions of the tests (Figure $3 b$ ).

\section{Relationship between DBS-related changes in motor symptoms and mood ratings}

Four patients were missing one of the UPDRS scores needed for the co-variate analysis, so the main analysis was repeated with $n=19$. The UPDRS change score was a significant covariate for or interacted significantly with all individual mood scales. Improvements in feeling strong $(\mathrm{F}(1,17)=0.11, \mathrm{p}=.74)$ and content $(\mathrm{F}(1,17)=0.43, \mathrm{p}=.52)$ with DBS ON vs OFF were no longer significant when changes in UPDRS-III were taken into account, but feeling better-coordinated ON stimulation remained significant $(\mathrm{F}(1,17)=10.37, \mathrm{p}=.005)$ even after covarying out the effect of improvement of motor symptoms $(\mathrm{F}(1,17)=4.75, \mathrm{p}=$. 044). Gregariousness was significantly lower when STN-DBS was ON than OFF $(F(1,17)=$ $15.85, \mathrm{p}=.001)$ when motor changes were included as a co-variate. With the UPDRS-III change score as a covariate, the combined factor of Contented-Discontented showed significant improvement with STN-DBS ON relative to $\mathrm{OFF}(\mathrm{F}(1,17)=8.82, \mathrm{p}=.009)$. Even after covarying UPDRS-III change scores, patients continued to report using less effort with STN-DBS ON than $\operatorname{OFF}(F(1,17)=4.58, \mathrm{p}=.047)$.

\section{Discussion}

On an established mood visual analogue scale (VAS) PD patients with bilateral STN-DBS reported themselves as better-coordinated, stronger and more contented with their stimulation ON than OFF. When combined into factors, the mood scores of the STN-DBS patients reflected greater alertness and calmness when ON stimulation. STN-DBS patients reported more positive mood on 14/16 mood VAS scales and that completion of cognitive and motor tests required less effort with stimulation ON than OFF. When improvements in motor symptoms were controlled for, STN-DBS patients reported feeling better-coordinated and more contented on the combined factors, but less gregarious (equivalent to more withdrawn) with DBS ON than OFF. Control PD patients reported no differences in mood, effort or fatigue on their two completions of the scales, indicating that there was no effect of completing the scales twice. 
These mood ratings are likely to reflect the patients' day-to-day mood states after STNDBS. Apart from the improved motor symptoms (discussed in the next paragraph), the 50\% reduction in dopaminergic medication post-surgery, which would have reduced ON-state involuntary dyskinesias, is likely to have contributed to the patients' reports of feeling better-coordinated and stronger with DBS ON. STN-DBS patients also reported feeling more contented with stimulation ON and reported that they needed less effort to complete the intervening cognitive tests, which is consistent with feeling stronger and better coordinated.

The analysis of covariance showed that the mood ratings were influenced by the improvement of the motor symptoms rated on the UPDRS-III. The significant stimulationinduced improvements in feeling strong and contented were largely accounted for by improved motor symptoms. Reports of feeling better co-ordinated and requiring less effort for task completion remained significant even after covarying out the UPRDS-III change score. Differences between DBS ON vs OFF became significant for the combined factor of Contented-Discontented, suggesting that patients' greater sense of contentment with stimulation $\mathrm{ON}$ had been masked by variance associated with changes in motor symptoms with stimulation. A decrease in gregariousness, equivalent to an increase in feeling withdrawn, also became significant after covarying out the UPDRS change score. This potentially reflects increased apathy with STN-DBS ON, as reported in other studies ${ }^{4}$. The apathy questionnaire used in the current study was only completed with STN-DBS ON, so cannot shed further light on this issue.

The results of the current study are consistent with the few previous studies of the short-term effects of STN-DBS on mood. Bilateral STN-DBS patients with stimulation ON reported less fatigue and mental confusion ${ }^{6}$, more activity and euphoria and less sedation and dysphoria $^{7}$, and had lower BDI and apathy scores ${ }^{8,18}$. Patients with unilateral STN-DBS reported less tiredness but more anger and confusion with stimulation $\mathrm{ON}^{5}$. All of these studies were done between 3 months and 4 years after surgery.

The VAS used by us has been validated ${ }^{9,10}$, was more comprehensive and had better face validity for the patients than measures employed in previous studies ${ }^{4,5,6,7,8,18}$. In addition to mood items, the VAS included somatic and alertness items which are relevant to the assessment of STN-DBS effects. We used an unoperated PD control group to ensure that changes in the VAS ratings for the STN-DBS group did not merely reflect the effect of two consecutive assessments. In contrast, the interpretation of the results of this study is limited by the small sample size and the relatively large range of intervals between surgery and testing. Mood may be more labile shortly after STN-DBS surgery ${ }^{3}$, and it is possible that our sample may be heterogenous in that respect, although the majority were assessed 6 months or more after surgery when transient mood changes would ordinarily have subsided. DBS can potentially have placebo effects as demonstrated for motor function ${ }^{19,20}$ which could also influence self-reported mood. While inclusion of the PD control group and counterbalancing of the ratings with DBS ON vs OFF in our study controlled for some confounding factors, placebo effects could have potentially influenced the results and should be addressed in future studies.

Short-term DBS ON versus OFF effects on mood resemble the levodopa-associated nonmotor fluctuations experienced by some PD patients who reported anxiety, irritability, fatigue and depression OFF medication which improved ON medication ${ }^{21,22}$. Some levodopa-induced non-motor fluctuations, particularly sensory, cognitive and autonomic fluctuations were alleviated with STN-DBS, whereas 'psychic' fluctuations were less consistently improved ${ }^{23}$. It is interesting to compare the effects of STN-DBS and levodopa on mood in PD, given the difference in localisation/selectivity of action of the two 
treatments. Both levodopa and STN-DBS improved mental confusion ${ }^{6}$, induced feelings of greater activity and euphoria and less dysphoria ${ }^{7}$, improved BDI scores ${ }^{8}$ and reduced apathy scores $^{18}$. Levodopa reduced 'feeling unsafe' ${ }^{6}$ and increased hedonia scores ${ }^{8}$, but STN-DBS had no effect. STN-DBS reduced fatigue ${ }^{6}$ and feelings of sedation ${ }^{7}$ while levodopa had no effect. The effects of levodopa in increasing positive mood and activity and reducing confusion are consistent with the known role of mesolimbic dopamine in enhancing reward and stimulus salience ${ }^{24,25}$. However, it is not known whether STN-DBS stimulation affects mesolimbic dopamine; and the improvement of mood and activity and reduction of confusion with STN-DBS may be mediated by stimulation of limbic STN regions leading to effects on limbic areas such as the anterior cingulate corte ${ }^{26}$.

The studies on the short-term effects of STN-DBS on mood indicate that stimulation has a small but generally positive effect on mood in patients for whom STN-DBS improves the motor symptoms of PD. The only point of concern is that STN-DBS patients reported feeling less gregarious (equivalent to more withdrawn) with stimulation ON, after controlling for improved motor symptoms, which may relate to reports of apathy after STNDBS. The patients in this and previous studies had had STN-DBS relatively recently, so it is not clear whether similarly positive overall short-term effects of stimulation on mood would be replicated 5 or 10 years after surgery. In the short-term our results are clinically useful for reassuring patients that switching STN-DBS on is likely to have mostly beneficial effects on mood and sense of well-being. However, these group effects may not apply to all individuals, as Czernicki et al. ${ }^{18}$ found individual differences in short-term STN-DBS effects on apathy. Furthermore, Okun et al. ${ }^{5}$ reported that unilateral stimulation more ventrally in the STN worsened mood, suggesting that electrode positioning in the STN may be important.

A better understanding of the short-term effects of STN-DBS stimulation on a wide range of mood states could contribute to improved clinical management of patients with STN-DBS and potentially to our understanding of the longer-term effects of DBS. A number of aspects require further study. The time course of the short-term effect of STN-DBS on mood is not known as all studies so far, including this one, have assessed patients with STN-DBS at a single time-point. There may be transient mood effects in the first weeks and months after STN-DBS surgery ${ }^{27}$, whereas slow development of longer-term mood problems may also occur $^{28}$. If the time-course of the short-term effects of STN-DBS on mood was known, it could perhaps be used as a predictor of longer-lasting mood problems after surgery, allowing pre-emptive treatment and giving some insight into the development of longer-term neural adaptation to STN-DBS. Evaluation of mood states with a strong motivational element, such as enthusiasm, interest and engagement, may be particularly important because of concerns about the longer-term association of STN-DBS with apathy ${ }^{28}$. Similarly, evaluation of the short-term effects of STN DBS on impulsivity may clarify impulsive decision-making reported with DBS ON versus $\mathrm{OFF}^{29}$ and the increased suicide risk following surgery ${ }^{30}$.

\section{Acknowledgments}

We are grateful to the PD patients who took part in this study. The research was supported by a postdoctoral fellowship from Parkinson's UK (LW) and an NIH R01 grant (NS40865-02; MJ).

\section{References}

1. Limousin P, Greene J, Pollak P, Rothwell J, Benabid AL, Frackowiak R. Changes in cerebral activity pattern due to subthalamic nucleus or internal pallidum stimulation in Parkinson's disease. Ann Neurol. 1997; 42:283-291. [PubMed: 9307248] 
2. Krack P, Poepping M, Weinert D, Schrader B, Deuschl G. Thalamic, pallidal, or subthalamic surgery for Parkinson's disease? J Neurol. 2000; 247(Suppl 2):II122-II134. [PubMed: 10991659]

3. Anderson KE, Mullins J. Behavioral changes associated with deep brain stimulation surgery for Parkinson's disease. Curr Neurol Neurosci Rep. 2003; 3:306-313. [PubMed: 12930700]

4. Drapier D, Peron J, Leray E, et al. Emotion recognition impairment and apathy after subthalamic nucleus stimulation in Parkinson's disease have separate neural substrates. Neuropsychologia. 2008; 46:2796-2801. [PubMed: 18579165]

5. Okun MS, Fernandez HH, Wu SS, et al. Cognition and mood in Parkinson's disease in subthalamic nucleus versus globus pallidus interna deep brain stimulation: the COMPARE trial. Ann Neurol. 2009; 65:586-595. [PubMed: 19288469]

6. Berney A, Panisset M, Sadikot AF, et al. Mood stability during acute stimulator challenge in Parkinson's disease patients under long-term treatment with subthalamic deep brain stimulation. Mov Disord. 2007; 22:1093-1096. [PubMed: 17394245]

7. Funkiewiez A, Ardouin C, Krack P, et al. Acute psychotropic effects of bilateral subthalamic nucleus stimulation and levodopa in Parkinson's disease. Mov Disord. 2003; 18:524-530. [PubMed: 12722166]

8. Witt K, Daniels C, Herzog J, et al. Differential effects of L-dopa and subthalamic stimulation on depressive symptoms and hedonic tone in Parkinson's disease. J Neuropsychiatry Clin Neurosci. 2006; 18:397-401. [PubMed: 16963590]

9. Norris $\mathrm{H}$. The action of sedatives on brain stem oculomotor systems in man. Neuropharmacology. 1971; 10:181-191. [PubMed: 5093959]

10. Bond A, Lader M. The use of analogue scales in rating subjective feelings. British Journal of Medical Psychology. 1974; 47:211-218.

11. Watson D, Tellegen A. Toward a consensual structure of mood. Psychol Bull. 1985; 98:219-235. [PubMed: 3901060]

12. Hall JC. Correlation of a modified form of Raven's progressive matrices (1938) with the Wechsler adult intelligence scale. Journal of Consulting Psychology. 1957; 21:23-26. [PubMed: 13406153]

13. Hart S, Smith CM, Swash M. Assessing intellectual deterioration. Br J Clin Psychol. 1986; 25 (Pt 2):119-124. [PubMed: 3730647]

14. Zigmond AS, Snaith RP. The hospital anxiety and depression scale. Acta Psychiatr Scand. 1983; 67:361-370. [PubMed: 6880820]

15. Starkstein SE, Mayberg HS, Preziosi TJ, Andrezejewski P, Leiguarda R, Robinson RG. Reliability, validity, and clinical correlates of apathy in Parkinson's disease. J Neuropsychiatry Clin Neurosci. 1992; 4:134-139. [PubMed: 1627973]

16. Tomlinson CL, Stowe R, Patel S, Rick C, Gray R, Clarke CE. Systematic review of levodopa dose equivalency reporting in Parkinson's disease. Mov Disord. 2010; 25:2649-2653. [PubMed: 21069833]

17. Williams-Gray CH, Foltynie T, Brayne CE, Robbins TW, Barker RA. Evolution of cognitive dysfunction in an incident Parkinson's disease cohort. Brain. 2007; 130:1787-1798. [PubMed: 17535834]

18. Czernecki V, Pillon B, Houeto JL, Welter ML, Mesnage V, Agid Y, Dubois B. Does bilateral stimulation of the subthalamic nucleus aggravate apathy in Parkinson's disease? J Neurol Neurosurg Psychiatry. 2005; 76:775-779. [PubMed: 15897497]

19. Pollo A, Torre E, Lopiano L, et al. Expectation modulates the response to subthalamic nucleus stimulation in Parkinsonian patients. NeuroReport. 2002; 13:1383-1386. [PubMed: 12167757]

20. De la Fuente-Fernández. Uncovering the hidden placebo effect in deep-brain stimulation for Parkinson's disease. Parkinsonism Relat D. 2004; 10:125-127.

21. Riley DE, Lang AE. The spectrum of levodopa-related fluctuations in Parkinson's disease. Neurology. 1993; 43:1459-1464. [PubMed: 8350996]

22. Witjas T, Kaphan E, Azulay JP, et al. Nonmotor fluctuations in Parkinson's disease: frequent and disabling. Neurology. 2002; 59:408-413. [PubMed: 12177375]

23. Witjas T, Kaphan E, Regis J, Jouve E, Cherif AA, Peragut JC, Azulay JP. Effects of chronic subthalamic stimulation on nonmotor fluctuations in Parkinson's disease. Mov Disord. 2007; 22:1729-1734. [PubMed: 17557355] 
24. Berridge KC, Robinson TE. What is the role of dopamine in reward: hedonic impact, reward learning, or incentive salience? Brain Res Brain Res Rev. 1998; 28:309-369. [PubMed: 9858756]

25. Schultz W. Getting formal with dopamine and reward. Neuron. 2002; 36:241-263. [PubMed: 12383780]

26. Alexander GE, DeLong MR, Strick PL. Parallel organization of functionally segregated circuits linking basal ganglia and cortex. Annu Rev Neurosci. 1986; 9:357-381. [PubMed: 3085570]

27. Houeto JL, Mesnage V, Mallet L, et al. Behavioural disorders, Parkinson's disease and subthalamic stimulation. J Neurol Neurosurg Psychiatry. 2002; 72:701-707. [PubMed: 12023409]

28. Thobois S, Ardouin C, Lhommée E, et al. Non-motor dopamine withdrawal syndrome after surgery for Parkinson's disease: predictors and underlying mesolimbic denervation. Brain. 2010; 133:1111-1127. [PubMed: 20237128]

29. Frank MJ, Samanta J, Moustafa AA, Sherman SJ. Hold your horses: impulsivity, deep brain stimulation, and medication in parkinsonism. Science. 2007; 318:1309-1312. [PubMed: 17962524]

30. Voon V, Krack P, Lang AE, et al. A multicentre study on suicide outcomes following subthalamic stimulation for Parkinson's disease. Brain. 2008; 131:2720-2728. [PubMed: 18941146] 
Figure 1a

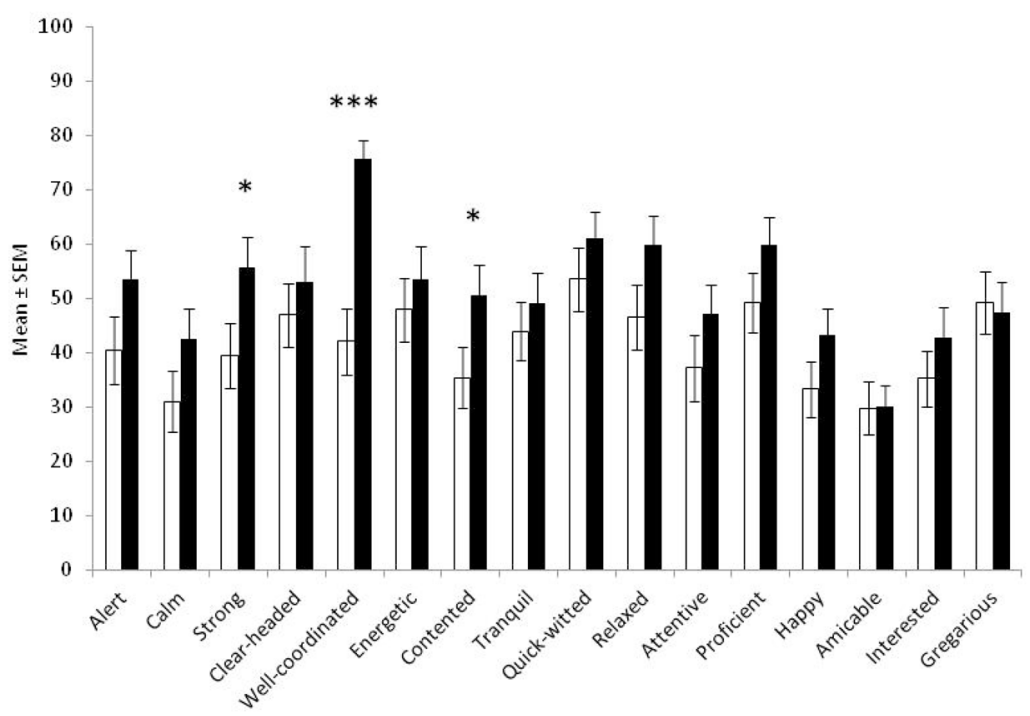

Figure 1b

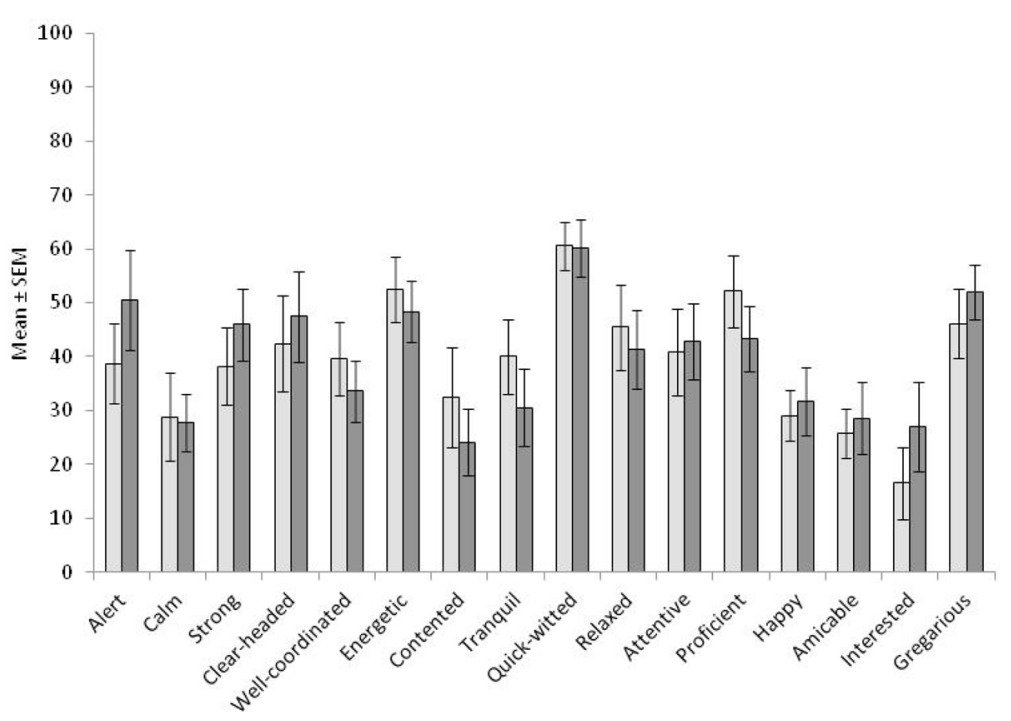

Figure 1.

(a) Mood ratings on visual analogue scales by PD patients with STN-DBS with stimulation ON (white bars) and OFF (black bars); (b) Mood ratings on visual analogue scales by PD controls $(\mathrm{n}=11)$, first time (light grey bars) and second time (dark grey bars). Note that lower scores represent more positive mood. $* \mathrm{p}<.05, * * * \mathrm{p}<.001$ comparison between ON and OFF STN-DBS for that mood scale. There were no significant differences between $1^{\text {st }}$ and $2^{\text {nd }}$ time for any mood scale for PD controls. Error bars are standard error of the mean. 


\section{Figure 2a}

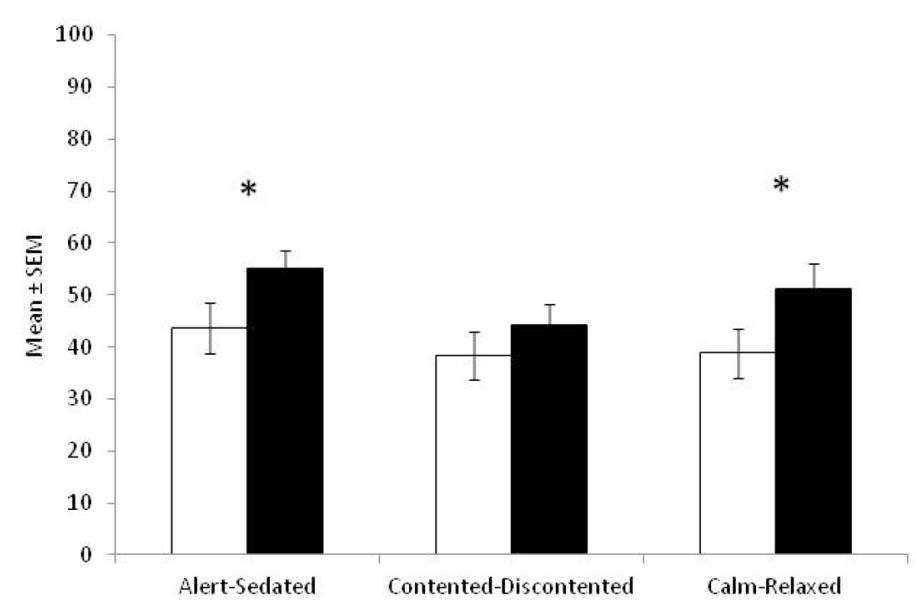

Figure 2b

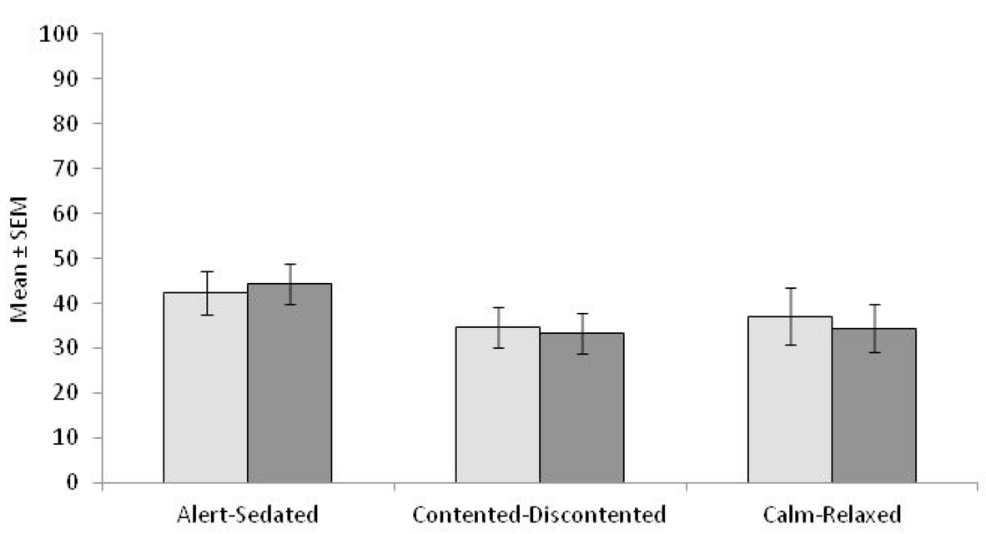

Figure 2.

Mood data for (a) STN-DBS patients ON (white bars) and OFF stimulation (black bars) and (b) PD controls completing the scale for the $1^{\text {st }}$ (light grey bars) and $2^{\text {nd }}$ time (dark grey bars) summed into the factors identified by Bond and $\operatorname{Lader}^{10} . * \mathrm{p}<.05$. Error bars are standard error of the mean. 


\section{Figure 3a}

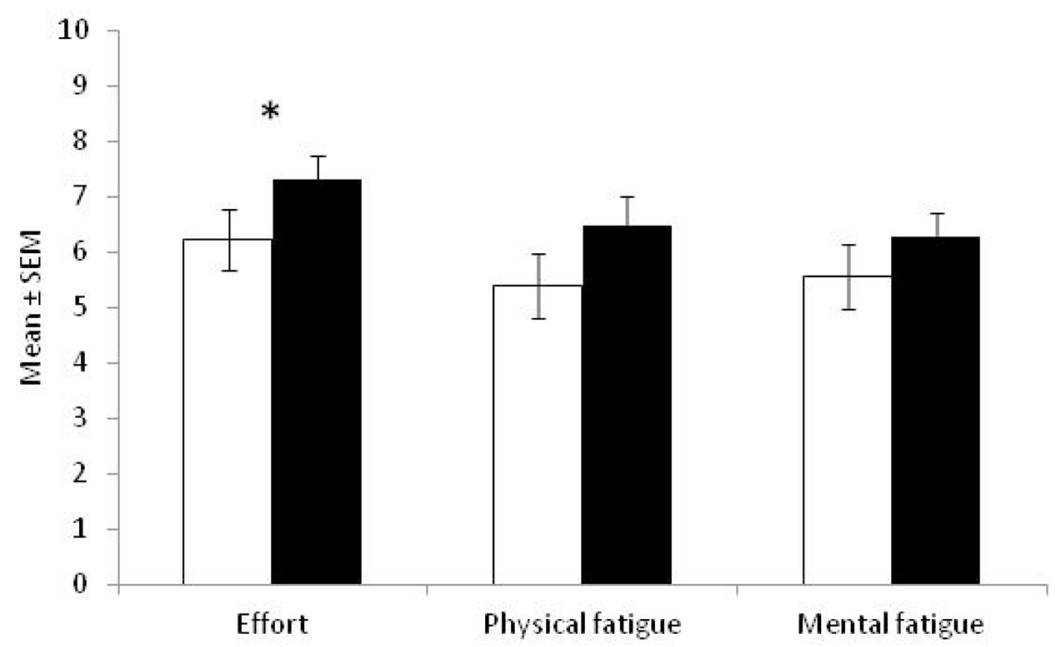

Figure 3b

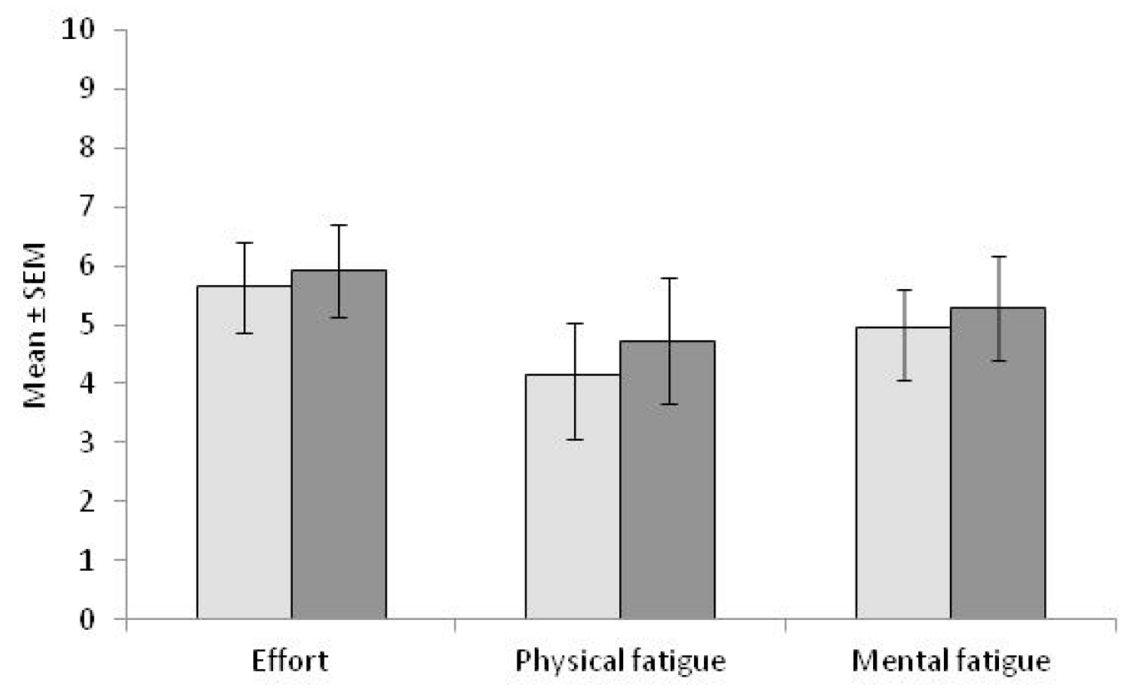

Figure 3.

Effort and Physical and Mental Fatigue ratings for (a) STN-DBS patients ON (white bars) and OFF stimulation (black bars) and (b) control PD patients for the $1^{\text {st }}$ (light grey bars) and $2^{\text {nd }}$ (dark grey bars) completion of the scales. Note that higher scores mean greater effort and fatigue. ${ }^{*} \mathrm{p}<.05$ comparison between ON and OFF STN-DBS for that scale. There were no significant differences between completing any of these scales for the $1^{\text {st }}$ and $2^{\text {nd }}$ time for PD controls. Error bars are standard error of the mean. 


\section{Table 1}

Clinical characteristics and demographic information for patients with Parkinson's disease (PD) who had deep brain stimulation of the subthalamic nucleus (STN-DBS) and for control PD patients.

\begin{tabular}{|c|c|c|c|}
\hline & PD STN-DBS & PD Controls & $p$-value \\
\hline$N$ & 23 & 11 & \\
\hline Age (years) & $55.9(6.3)$ & $60.5(6.1)$ & .053 \\
\hline Gender & 16 men & 7 men & 1.0 \\
\hline Handedness & 19 right-handed & 11 right-handed & .28 \\
\hline Education (years) & $13.6(2.7)$ & $14.8(4.6)$ & .35 \\
\hline Duration of illness (years) & $14.9(5.0)$ & $10.5(4.6)$ & .022 \\
\hline IQ estimate (Raven's Advanced Matrices) & $117.7(12.2)$ & $121.9(6.0)$ & .051 \\
\hline IQ - NART & $116.4(9.6)$ & $114.7(12.4)$ & .45 \\
\hline Apathy (ON stim for DBS) & $13.0(11.6) \mathrm{N}=11$ & $10.3(6.4)$ & .16 \\
\hline HAD-A (ON stim for DBS) & $4.9(3.6)$ & $6.9(3.3)$ & .50 \\
\hline HAD-D (ON stim for DBS) & $7.0(4.4)$ & $5.1(2.7)$ & .13 \\
\hline \multicolumn{4}{|l|}{ UPDRS-III } \\
\hline Pre-surgery ON medication & $9.9(6.4)$ & - & \\
\hline Pre-surgery OFF medication & $45.6(12.0)$ & - & \\
\hline \multicolumn{4}{|l|}{ At assessment } \\
\hline ON medication & - & $17.8(9.5)$ & \\
\hline OFF medication & - & $39.8(18.8)$ & \\
\hline OFF stim OFF medication & $45.6(19.0)$ & - & \\
\hline OFF stim ON medication & $18.2(6.6)$ & - & \\
\hline ON stim OFF medication & $22.2(12.5)$ & - & \\
\hline ON stim ON medication & $9.1(4.5)$ & - & \\
\hline \multicolumn{4}{|l|}{ Hoehn and Yahr } \\
\hline At assessment - best & $2.2(1.0)$ & $2.4(0.7)$ & .32 \\
\hline At assessment - worst & $3.3(1.0)$ & $3.1(1.1)$ & .77 \\
\hline \multicolumn{4}{|l|}{ Medication (L-DOPA equivalent, mg/day) } \\
\hline Pre-surgery & $1475.8(568.5)$ & - & \\
\hline At assessment & $735.5(341.8)$ & $841.0(496.5)$ & .26 \\
\hline \multicolumn{4}{|l|}{ Stimulation parameters } \\
\hline Frequency $(\mathrm{Hz})$ & $142.5(20.9)$ & - & \\
\hline Pulse width ( $\mu$ sec) & $63.8(10.0)$ & - & \\
\hline Intensity (V) & $3.1(0.7)$ & - & \\
\hline
\end{tabular}

